\title{
Exploring Religiosity and Spirituality in the Sexual Decision-Making of Black Gay and Bisexual Men
}

\author{
Derek T. Dangerfield II. ${ }^{1}$. Jeffery E. Williams ${ }^{2}$ - Alágra S. Bass ${ }^{3}$. \\ Timothy Wynter ${ }^{2} \cdot$ Ricky N. Bluthenthal ${ }^{2}$
}

Published online: 3 June 2019

๑) Springer Science+Business Media, LLC, part of Springer Nature 2019

\begin{abstract}
Many Black gay and bisexual men (BGBM) practice consistent religious worship and spirituality in order to cope with social challenges. This study utilized data from in-depth qualitative interviews conducted to explore personal meanings of religiosity and spirituality and how these constructs affect partner choices and sexual behaviors among BGBM. Interviews were conducted with 26 BGBM in Los Angeles, California, from September to November 2015. In-depth interviews yielded themes related to BGBM's understanding of religiosity and spirituality and how their personal beliefs influenced their partner choices and sexual risks. Implications for church-based and community-based interventions are discussed.
\end{abstract}

Keywords Black gay and bisexual men $\cdot$ Religion $\cdot$ Spirituality $\cdot$ Health

\section{Introduction}

In the USA, Black gay and bisexual men (BGBM) continue to be disproportionately impacted by HIV and sexually transmitted infections (STIs) (Centers for Disease Control and Prevention [CDC] 2015; Chan et al. 2017; Hess et al. 2017). Both religion and spirituality are salient for BGBM, who are more likely than other racial/ethnic groups of men who have sex with men (MSM) to report that religion and spirituality are very important (O'Leary et al. 2007). Although concepts of religiosity and spirituality overlap, religiosity is referred to as more structured, behavioral, and extrinsic, while spirituality is described as more subjective,

Derek T. Dangerfield II.

ddanger2@jhu.edu

1 Johns Hopkins School of Nursing, 855 N. Wolfe St Suite 601, Baltimore, MD 21205, USA

2 Department of Preventive Medicine, Keck School of Medicine, University of Southern California, Los Angeles, CA, USA

3 School of Social Work, University of Maryland, Baltimore, MD, USA 
internal, emotional, and intrinsic (Horn et al. 2005; Ironson et al. 2006; Johnstone et al. 2009). Many BGBM view religious worship and spirituality as deeply intertwined with family and community life and use religious practices to cope with social stressors and identity development challenges (Balaji et al. 2012; Foster et al. 2011; Quinn and Dickson-Gomez 2016). Clarifying the distinctions and similarities between the two concepts is important, and understanding the role of religion and spirituality in the lives of BGBM could provide insight into ways to improve sexual health for this group.

Prior studies show a paradox of religiosity and spirituality being associated with both risk of and protection against HIV and STIs among BGBM. Specifically, religiosity has been associated with being HIV-negative and protective against participating in condomless receptive anal intercourse (RAI) (Watkins et al. 2016a); interactions with religious family and churches are sources of support for some BGBM, even after sharing their sexuality or HIV status (Jeffries IV et al. 2014). Higher scores of spirituality have been associated with fewer condomless anal sex acts, lower levels of condomless casual sex, and lower substance use (Murray et al. 2007; Watkins et al. 2016a). However, religiosity and spirituality have also been associated with HIV/STI infection, illicit drug use (Watkins et al. 2016a), lower gay affirmation (Smallwood et al. 2015), and increased numbers of sexual partners (Nelson et al. 2017). Whether religiosity and spirituality are more protective or risky requires clarification (Watkins et al. 2016a, b). Some urban church leaders are open to balancing traditional theology with practical public health approaches to HIV prevention such as HIV testing and integrating prevention topics in sermons as social justice (Cunningham et al. 2011; Derose et al. 2016; Nunn et al. 2012; Wilson et al. 2011). However, more detailed information is needed about how religiosity or spirituality is constructed among BGBM and how those constructs influence sexual decision-making and risks.

Despite the attention given to reduce HIV and improve health outcomes among BGBM, religiosity and spirituality are two important cultural factors that remain understudied and underutilized. Cognitive dissonance and intersectionality are useful frameworks to understand the role of religion and spirituality in sexual risk for BGBM (Bowleg et al. 2013; Watkins et al. 2016a, b; Quinn and Dickson-Gomez 2016). Cognitive dissonance refers to conflicting attitudes, beliefs, or behaviors that lead to feelings of discomfort. Intersectionality refers to the cumulative experiences of different forms of discrimination due to having multiple, marginalized social identities (e.g., being a Black and gay/bisexual man in the USA) (Bowleg et al. 2013; Quinn and Dickson-Gomez 2016; Warner and Shields 2013). BGBM often experience cognitive dissonance due to an intersecting conflict between their sexual orientation and their religious upbringing (Balaji et al. 2012; Hill and McNeely 2013; Quinn and Dickson-Gomez 2016; Watkins 2016a). For Black Americans, churches have been central in setting norms and defining values (Smith et al. 2005) and have provided refuge from racial discrimination and marginalization (Lincoln and Mamiya 1990). However, both the independence and interplay of race and sexual orientation among BGBM could have implications for HIV/STI risk or safety (Bowleg et al. 2013; Warner and Shields 2013). 
While studies have described the role of the church and ways in which religion and spirituality influence sexual risks, few have explored how BGBM reconcile their intersectional identities as religious/spiritual/Black/sexual minority men (Pitt 2010; Winder 2015), or how their religious or spiritual views affect sexual decision-making. Given the importance of religion and spirituality in many BGBM's lives and the need for culturally appropriate interventions to address HIV vulnerability within this population, more information about the role of religiosity and spirituality in the sexual lives of BGBM is still needed. Obtaining an in-depth narrative on these concepts could inform culturally appropriate strategies to mitigate HIV/STI risk among this population (Wilson et al. 2016). As such, this study describes the personal meanings of religiosity and spirituality as reported by BGBM and how these constructs affect their partner choices and sexual behaviors.

\section{Methods}

This study utilized data from in-depth qualitative interviews conducted as part of a larger study that explored generational differences and developmental pathways to sexual risk among BGBM (Dangerfield et al. 2017a). Interviews were conducted with 26 BGBM in Los Angeles, California, from September to November 2015. Eligibility was based upon the following criteria: self-identifying as Black or AfricanAmerican, being $\geq 18$ years of age, and reporting oral or anal sex practice in the previous 6 months with at least one male partner. Interviews probed domains related to the meaning of religiosity and spirituality, men's experiences in churches, and how their religious or spiritual beliefs affected their sexual behaviors (i.e., partner selection, sexual positioning practices, and condom use). Interviews ranged from $55 \mathrm{~min}$ to $1.5 \mathrm{~h}$. Study procedures were reviewed and approved by the University of Southern California Health Sciences Campus Institutional Review Board.

\section{Qualitative Data Analysis}

Detailed description of data analysis is published (Dangerfield et al. 2017a). Briefly, interviews were audio-recorded and transcribed verbatim. Two coders read all transcripts to identify overarching themes, looked for repetitions across interviews, and then developed a codebook. Themes were identified as either patterns that were associated with specific questions, or expressions that provided examples of questions and concepts (Denzin and Lincoln 2011; Ober et al. 2017; Ryan and Bernard 2003). Transcript coding was conducted using Atlas.ti 7.1 (2016). Areas of text related to each domain were coded independently by the two coders, and inter-rater reliability (IRR) was measured to ensure consistency between transcript coding (kappa $\geq 0.70$ ). IRR was measured twice to confirm coding consistency; inconsistencies were reviewed and showed minor differences in the lengths of quoted text but not overall text quality. Data presented here represent the range of themes related to the meanings of religiosity and spirituality and how personal views of religiosity and spirituality affected sexual behaviors as described by BGBM. 


\section{Results}

The age of participants ranged from 24 to 61 years. In the demographic survey, $69 \%$ of participants identified as gay; the remaining 31\% identified as bisexual. Most $(19,73 \%)$ were single/not in a committed relationship. Seven $(27 \%)$ self-reported being HIV positive. Sixteen participants self-identified as "spiritual," two referred to themselves as "religious," and five self-identified as both "spiritual" and "religious," some of whom mentioned having leanings that were "more spiritual." Two identified as "neither spiritual nor religious"; one of those expressed that he did "believe in God." In-depth interviews yielded themes related to BGBM' understanding of religiosity and spirituality and how their personal beliefs influenced their partner choices and sexual risk behaviors.

\section{Emerging Themes}

\section{Defining Religious Versus Spiritual Identity}

Participants were asked broadly to indicate whether they identified as either "religious" or "spiritual." Across interviews, BGBM expressed similar distinctions between the meanings of religiosity and spirituality. Some shared that being "religious" referred to a set of practices or strict rules that were prescribed by society, the church, or the Bible. They also expressed that identifying as spiritual was a more independent, personal connection with a "higher power" that was not bound by specific rules. One participant, who identified as both religious and spiritual, said that there was no difference between being spiritual and religious, but alluded to an independent, personal connection to God in a way that others described as being spiritual. Of note, one man who identified as "neither religious nor spiritual" mentioned a series of practices that helped him maintain sobriety that resembled spiritual/religious activities. When asked whether he identified as either religious or spiritual, he said,

No, but I pray and meditate, I go to recovery groups so it helps me to stay grounded, meditation, relaxation, you know, things like that. And I live by myself, so that's one of the things that, you know, helps me. (Participant 013)

Although he did not consider himself spiritual or religious, his practices were conceptually similar to the descriptions others provided.

Participants also reflected upon how personal development helped them reconcile conflicting feelings of same-sex attractions as sinful, while still wanting to engage in spiritual or religious activities. They suggested that engaging in "spirituality" rather than "religiosity" helped to reconcile this cognitive dissonance and to cope with judgmental practices within the Black or church community. BGBM who identified as "spiritual" distanced themselves from the church because of this conflict, even though for many it was a source of support in some ways and the foundation of their personal values. 


\section{Choosing Partners}

Interviews also yielded a theme about how religion and spirituality influenced BGBM's choices of sexual partners, regardless of religious or spiritual identification. Some mentioned how their religious/spiritual identity made them "want to change my life because that's in the Bible it's (homosexuality) against God's will" (Participant 05, Religious). One participant mentioned that he chose to engage in sexual relationships with women because of his spiritual/religious conflict. When asked about his interest in male and female partners, he said, "The Christian thing is kind of swaying into that right now... you know, my brother, he's full-fledged. He don't like women at all. I'm bi...I just ask God to forgive me, you know, and I believe he will" (Participant 003, Spiritual). Some men who identified as gay shared how their religious/spiritual identity also affected the way they selected male sexual partners. Specifically, that they would not choose "feminine" male partners because of their upbringing in the church. One man put it this way,

I won't pick... a flaming guy, you know, and if he's too flamboyant I don't even want to be around him. He can't come to my spot... I feel more comfortable with a person that [is] like me, they wouldn't know [if] he's gay. (Participant 020, Spiritual)

Across religious and spiritual identification, BGBM's partner selections were impacted.

\section{Sexual Activity and Risk}

BGBM also shared how religious and spiritual identity influenced sexual behaviors. Some men mentioned how having a spiritual foundation prevented them from engaging in sexual intercourse and condomless sex. Specifically, one man said,

I don't feel like religion too much affects my sexual relationship, because if it did I probably wouldn't be having sex. As far as spiritual, I feel like it affects me because it kind of grounds me and kind of gives me reasons to be safe or love myself more because I don't want to be hurt, I don't want to be sick... (Participant 002, Religious \& Spiritual 002)

Another said, "I feel like, I mean, that doesn't always happen but I feel like sex is something sacred to me and I'm not fixin' to be out having sex with like just random people, and not talk to them again" (Participant 024, Spiritual). These kinds of comments captured how men viewed their sexual activities relative to their spiritual values. However, of note, many who identified as "spiritual" mentioned wanting monogamous male partnerships, but their practices were not always congruent with their ideal values.

Conversely, some mentioned how identifying as "spiritual" rather than "religious" allowed them to explore their sexuality, which occasionally led to sexual 
risks. They shared that after moving away from being religious and identifying as spiritual, they were able to explore their sexuality with less shame, be open to having male partners, and explore their sexuality. When asked whether spiritual identification influenced sexual risk taking, this participant, who described habits of condomless IAI and RAI with partners while using drugs, said, "Kind of. It leaves me open to like, you know just exploring the realm of anything and like everything because I'm like always open to try new things, new experiences, like whatever, so, yeah" (Participant 010, Spiritual). Statements like this reveal how spirituality and connecting with partners could increase condomless sex. However, having this kind of spiritual freedom could increase sexual risk.

\section{Sexual positioning practices}

Across religious and spiritual self-identities, BGBM also mentioned how those concepts impacted sexual positioning practices. Some mentioned how spirituality liberated them to be open to both receptive and insertive anal intercourse (IAI) practices. When discussing the relationship between his beliefs and partner preferences, one man talked about it this way, "I already told the universe that I wanted this at the same time, I said 'Allow me to be an adult be able to handle life on my own ...' Yeah so where we top and bottom each other" (Participant 024, Spiritual). Other participants who identified as spiritual shared that their beliefs helped them be more selective, particularly in their decision to engage in RAI. One participant detailed how he was selective in his decision to practice RAI.

...it goes back to my spiritual self, and it just goes back to I'm giving you something that I cannot take back, and that I want to feel secure what I'm giving to you, and I want to give you all of me, and we make a connection. Because I feel like sexual experiences are connections...like if we're not connected and we're not feeling like nothing else in the world is mattering right and that we're out of our element, then it's kind of a waste... (Participant 002, Religious and Spiritual)

Condom use did not emerge as a component affecting these views related to sexual positioning.

\section{Discussion}

The present study explored the descriptions of religiosity and spirituality as reported by BGBM and how these concepts affect partner choices and sexual behaviors. Findings showed how BGBM renounced religiosity and/or church affiliation to become what they understand is spiritual. However, BGBM held values and beliefs similar to their religious upbringing and shared stories of internal conflict and patterns of partner selection, sexual decision-making, and sexual risk. We found that religiosity and spirituality impacted the selection of sexual partners for some BGBM and a novel concept that these constructs also impacted sexual positioning practices. 
Data from the present study are congruent with other research among BMSM that describes how some are disconnected from their Christian upbringings but still accept some of the religious tenets of their youth, and practice religion differently as adults or abandon religious participation altogether (Jeffries IV et al. 2014; Winder 2015). Our data suggests that identifying as "spiritual" enabled BGBM to maintain aspects of their upbringing in the church and provided a freedom to accept themselves as same-sex attracted men.

Our finding that religiosity and spirituality affect sexual positioning practices for BGBM is novel. It is important to consider the role of religion/spirituality in sexual behaviors since risk of HIV and sexually transmitted infections vary by sexual positioning (Dangerfield et al. 2017b; Patel et al. 2014). Practicing both insertive and RAI with male partners increases HIV and extragenital STI risk among MSM (Dangerfield et al. 2017b). However, more research is needed to understand the relationship between religious/spiritual values, sexual positioning, and condom use among BGBM. Prevention messaging should be careful not to provide stigmatizing messages about RAI with male partners. From these data, condom use did not emerge as a "mediator" between spiritual/religious views and sexual positioning; however, additional research should target this relationship for study.

Although same-sex marriage is available and might reflect a more tolerant society, BGBM in this study still struggled with feelings of internalized homonegativity from their upbringing in churches (arguably within Black communities) that would not allow them to normalize their same-sex attractions. Other research has described how BMSM perceive their behaviors as "sinful" (Glick and Golden 2010; Jeffries et al. 2008, 2014; Nelson et al. 2017; Valera and Taylor 2011; Winder 2015). It is imperative to recognize the lasting impression that homonegativity in church or other religious communities has on BMSM and their perceptions of themselves. For many BGBM, the feelings of shame originate from being members of a church and the Black community and remain salient in their identities. This stigma facilitates sexual risks for BMSM and decreases the efficacy of HIV prevention work (Watkins et al. 2016a). Specifically, stigma discourages BMSM from establishing long-term, monogamous relationships with men, seeking prevention supplies (i.e., condoms and lubricant), and engaging in safer sex practices such as condom use (Foster et al. 2011; Quinn and Dickson-Gomez 2016; Murray et al. 2007; Watkins et al. 2016a).

Many religious organizations have initiated interventions in response to the HIV epidemic, but with limited success (Derose et al. 2016). HIV awareness and screening interventions indicate that most Black church leaders believe that the church should talk about HIV and promote HIV testing (Berkley-Patton et al. 2010; Wilson et al. 2011). When implementing church-based educational interventions, including community-based participatory research (CBPR), peer leadership workshops, pastor-delivered sermons on HIV, and HIV-testing events in various settings, there are increases in sexual health knowledge, perceived risk of acquiring HIV, and HIV-testing rates (Berkley-Patton et al. 2010; Derose et al. 2016; Griffith et al. 2010). Several barriers exist in engaging faith-based leaders in HIV education and prevention. Some religious leaders express concerns in endorsing extramarital sex by promoting condom use, have limited educational information appropriate for a faith-based audience, and have a fears of losing 
congregants and revenue by discussing sexuality (Nunn et al. 2012). Furthermore, some churches are hesitant to engage in HIV prevention efforts given their beliefs about same-sex behavior (Bryant-Davis et al. 2016; Pingel and Bauermeister 2018; Wilson et al. 2011). These hesitations often give rise to stigma and places BGBM at risk of social isolation and significant stress that may diminish the mental and physical health benefits generally associated with religiosity (BryantDavis et al. 2016; Pingel and Bauermesiter 2017).

It is also interesting to note these findings among BGBM in Los Angeles, where the social climate is more liberal and gay-friendly than other parts of the USA, for example, southern cities with majority Black populations (Valera and Taylor 2011). Despite living in a "more liberal" social context, BGBM in our study still reported similar dissonance and consequences of their intersectionality as Black/gay/bisexual men, which does not affect MSM of other ethnicities in the same way (Hill and McNeely 2013). This intersectionality could create similar outcomes as those that exist in a less tolerant city.

There are limitations to consider with this study. The study sample size was limited to participants in Los Angeles and was not representative of all BGBM; many were low income and substance involved; all of the men in this study identified as either gay or bisexual. Additionally, the constructs of religiosity and spirituality were open-ended qualitative questions; therefore, we cannot quantify the role of these constructs on participants' behaviors.

More work is needed to understand the concepts and construction of religiosity and spirituality for BGBM, and how BGBM might use spirituality as a proxy for religiosity and coping. Understanding these concepts as defined by BMSM could aid in designing culturally congruent HIV prevention and sexual health programs (Foster et al. 2011) that are community based, particularly since many of these individuals mentioned discontinued church participation. Community-based interventions could incorporate faith- and spirituality-based messaging to increase HIV prevention and reduce risk behaviors, including condomless sexual activity and drug use (Winder 2015). Interventions should also consider the role of sexual positioning; however, it is important to balance a harm reduction narrative that does not create sexuality stigma for MSM. Our findings suggest that focus on the connection experienced with "a higher power," the "spiritual self," and personal values could be informative in a culturally congruent intervention that incorporates spiritual values for BGBM. Additionally, interventions should begin to target ways to combat long-term experiences of homonegativity and stigma from churches among BMSM. Since experiences of homonegativity and stigma play an important role in the HIV disparities between MSM groups in the USA (Maulsby et al. 2013) and since spirituality and religious values remain for BMSM, it is important to find ways to integrate positive spiritual messages for BMSM. Our data show that the intersection of being Black, gay/bisexual, and religious creates a problem, so within that intersection we must find the solution.

Funding This study was funded by the National Science Foundation Graduate Research Fellowship Program. 


\section{Compliance with Ethical Standards}

Conflict of interest The authors declare that they have no conflict of interests.

Human and Animal Rights All procedures performed in studies involving human participants were in accordance with the ethical standards of the institutional and/or national research committee and with the 1964 Helsinki Declaration and its later amendments or comparable ethical standards.

\section{References}

Balaji, A. B., Oster, A. M., Viall, A. H., Heffelfinger, J. D., Mena, L. A., \& Toledo, C. A. (2012). Role flexing: How community, religion, and family shape the experiences of young Black men who have sex with men. AIDS Patient Care and STDs, 26(12), 730-737. https://doi.org/10.1089/ apc.2012.0177.

Berkley-Patton, J., Bowe-Thompson, C., Bradley-Ewing, A., Hawes, S., Moore, E., Williams, E., et al. (2010). Taking it to the pews: A CBPR-guided HIV awareness and screening project with black churches. AIDS Education and Prevention, 22(3), 218-237.https://doi.org/10.1521/ aeap.2010.22.3.218.

Bowleg, L., Teti, M., Malebranche, D. J., \& Tschann, J. M. (2013). "It's an uphill battle everyday": Intersectionality, low-income Black heterosexual men, and implications for HIV prevention research and interventions. Psychology of Men \& Masculinity, 14(1), 25-34. https://doi. org/10.1037/a0028392.

Bryant-Davis, T., Ellis, M. U., Edwards, N., Adams, T. P., Counts, P., Arline-Bradley, S., et al. (2016). The role of the Black church in HIV prevention: Exploring barriers and best practices. Journal of Community \& Applied Social Psychology, 26(5), 388-408. https://doi.org/10.1002/casp.2270.

Centers for Disease Control and Prevention. (2015). African American|Gay and Bisexual Men|HIV by Group|HIV/AIDSICDC. January 12, 2016, from http://www.cdc.gov/hiv/group/msm/bmsm.html.

Chan, G. A., Johnson, K. L., Mosca, N. G., Dobbs, T. E., Dombrowski, J. C., Bennett, A. B., et al. (2017). Emerging regional and racial disparities in the lifetime risk of human immunodeficiency virus infection among men who have sex with men: A comparative life table analysis in King County. WA and Mississippi. Sexually Transmitted Diseases, 44(4), 227-232. https://doi.org/10.1097/OLQ.00000 00000000589.

Cunningham, S. D., Kerrigan, D. L., McNeely, C. A., \& Ellen, J. M. (2011). The role of structure versus individual agency in churches' responses to HIV/AIDS: A case study of Baltimore City Churches. Journal of Religion and Health, 50(2), 407-421. https://doi.org/10.1007/s10943-009-9281-7.

Dangerfield, D. T., II, Smith, L. R., Anderson, J. N., Bruce, O. J., Farley, J., \& Bluthenthal, R. (2017a). Sexual positioning practices and sexual risk among Black gay and bisexual men: A life course perspective. AIDS and Behavior, 22(6), 1919-1931. https://doi.org/10.1007/s10461-017-1948-6.

Dangerfield, D. T., II, Smith, L. R., Williams, J., Unger, J., \& Bluthenthal, R. (2017b). Sexual positioning among men who have sex with men: A narrative review. Archives of Sexual Behavior, 46(4), 869-884. https://doi.org/10.1007/s10508-016-0738-y.

Denzin, N. K., \& Lincoln, Y. S. (2011). The SAGE handbook of qualitative research. Thousand Oaks: SAGE.

Derose, K. P., Griffin, B. A., Kanouse, D. E., Bogart, L. M., Williams, M. V., Haas, A. C., et al. (2016). Effects of a pilot church-based intervention to reduce HIV stigma and promote HIV testing among African Americans and Latinos. AIDS and Behavior, 20(8), 1692-1705. https://doi.org/10.1007/ s10461-015-1280-y.

Foster, M. L., Arnold, E., Rebchook, G., \& Kegeles, S. M. (2011). 'It's my inner strength': Spirituality, religion and HIV in the lives of young African American men who have sex with men. Culture, Health \& Sexuality, 13(9), 1103-1117. https://doi.org/10.1080/13691058.2011.600460.

Glick, S. N., \& Golden, M. R. (2010). Persistence of racial differences in attitudes toward homosexuality in the United States. Journal of Acquired Immune Deficiency Syndromes, 55(4), 516-523. https:// doi.org/10.1097/QAI.0b013e3181f275e0. 
Griffith, D. M., Pichon, L. C., Campbell, B., \& Allen, J. O. (2010). YOUR blessed health: A faith-based CBPR approach to addressing HIV/AIDS among African Americans. AIDS Education and Prevention, 22(3), 203-217. https://doi.org/10.1521/aeap.2010.22.3.203.

Hess, K. L., Hu, X., Lansky, A., Mermin, J., \& Hall, H. I. (2017). Lifetime risk of a diagnosis of HIV infection in the United States. Annals of Epidemiology, 27(4), 238-243. https://doi.org/10.1016/j. annepidem.2017.02.003.

Hill, W. A., \& McNeely, C. (2013). HIV/AIDS disparity between African-American and Caucasian men who have sex with men: Intervention strategies for the black church. Journal of Religion and Health, 52(2), 475-487. https://doi.org/10.1007/s10943-011-9496-2.

Horn, M., Piedmont, R., Fialkowski, G., Wicks, R., \& Hunt, M. (2005). Sexuality and Spirituality: The Embodied Spirituality Scale. Theology \& Sexuality, 12(1), 81-101. https://doi.org/10.1177/13558 35805057788.

Ironson, G., Stuetzle, R., \& Fletcher, M. A. (2006). An increase in religiousness/spirituality occurs after HIV diagnosis and predicts slower disease progression over 4 years in people with HIV. Journal of General Internal Medicine, 21(Suppl 5), S62-S68. https://doi.org/10.1111/j.1525-1497.2006.00648 .x.

Jeffries, W. L., Dodge, B., \& Sandfort, T. G. M. (2008). Religion and spirituality among bisexual Black men in the USA. Culture, Health \& Sexuality, 10(5), 463-477. https://doi.org/10.1080/1369105070 1877526.

Jeffries, W. L., IV, Okeke, J. O., Gelaude, D. J., Torrone, E. A., Gasiorowicz, M., Oster, A. M., et al. (2014). An exploration of religion and spirituality among young, HIV-infected gay and bisexual men in the USA. Culture, Health \& Sexuality, 16(9), 1070-1083. https://doi.org/10.1080/13691 058.2014.928370.

Johnstone, B., Yoon, D. P., Franklin, K. L., Schopp, L., \& Hinkebein, J. (2009). Re-conceptualizing the factor structure of the brief multidimensional measure of religiousness/spirituality. Journal of Religion and Health, 48(2), 146. https://doi.org/10.1007/s10943-008-9179-9.

Lincoln, C. E., \& Mamiya, L. H. (1990). The Black Church in the African American Experience. Duke University Press.

Maulsby, C., Millett, G., Lindsey, K., Kelley, R., Johnson, K., Montoya, D., et al. (2013). HIV among Black men who have sex with men (MSM) in the United States: A review of the literature. AIDS and Behavior, 18(1), 10-25. https://doi.org/10.1007/s10461-013-0476-2.

Murray, K. M., Ciarrocchi, J. W., \& Murray-Swank, N. A. (2007). Spirituality, religiosity, shame and guilt as predictors of sexual attitudes and experiences. Journal of Psychology and Theology, 35(3), 222.

Nelson, L. E., Wilton, L., Zhang, N., Regan, R., Thach, C. T., Dyer, T. V., et al. (2017). Childhood Exposure to Religions With High Prevalence of Members Who Discourage Homosexuality Is Associated With Adult HIV Risk Behaviors and HIV Infection in Black Men Who Have Sex With Men. American Journal of Men's Health, 11(5), 1309-1321. https://doi.org/10.1177/1557988315626264.

Nunn, A., Cornwall, A., Chute, N., Sanders, J., Thomas, G., James, G., et al. (2012). Keeping the faith: African American faith leaders' perspectives and recommendations for reducing racial disparities in HIV/AIDS infection. PLoS One, 7(5), e36172. https://doi.org/10.1371/journal.pone.0036172.

O’Leary, A., Fisher, H. H., Purcell, D. W., Spikes, P. S., \& Gomez, C. A. (2007). Correlates of risk patterns and race/ethnicity among HIV-positive men who have sex with men. AIDS and Behavior, 11(5), 706-715. https://doi.org/10.1007/s10461-006-9205-4.

Ober, A. J., Dangerfield, D. T., II, Shoptaw, S., Ryan, G., Stucky, B., \& Friedman, S. R. (2017). Using a "positive deviance" framework to discover adaptive risk reduction behaviors among high-risk HIVnegative Black men who have sex with men. AIDS and Behavior, 22, 1-14.

Patel, P., Borkowf, C. B., Brooks, J. T., Lasry, A., Lansky, A., \& Mermin, J. (2014). Estimating peract HIV transmission risk: A systematic review. AIDS, 28(10), 1509-1519. https://doi.org/10.1097/ QAD.0000000000000298.

Pingel, E. S., \& Bauermeister, J. A. (2018). 'Church hurt can be the worst hurt': Community stakeholder perceptions of the role of Black churches in HIV prevention among young Black gay and bisexual men. Culture, Health \& Sexuality, 20(2), 218-231. https://doi.org/10.1080/13691058.2017.13387 56.

Pitt, R. N. (2010). "Killing the messenger”: Religious Black gay men's neutralization of anti-gay religious messages. Journal for the Scientific Study of Religion, 49(1), 56-72. https://doi.org/10.111 1/j.1468-5906.2009.01492.x. 
Quinn, K., \& Dickson-Gomez, J. (2016). Homonegativity, religiosity, and the intersecting identities of young Black men who have sex with men. AIDS and Behavior, 20(1), 51-64. https://doi. org/10.1007/s10461-015-1200-1.

Ryan, G. W., \& Bernard, H. R. (2003). Techniques to identify themes. Field Methods, 15(1), 85-109. https://doi.org/10.1177/1525822X02239569.

Smallwood, S. W., Spencer, S. M., Ingram, L. A., Thrasher, J. F., \& Thompson-Robinson, M. V. (2015). Examining the relationships between religiosity, spirituality, internalized homonegativity, and condom Use among African American men who have sex with men in the Deep South. American Journal of Men's Health. https://doi.org/10.1177/1557988315590835.

Smith, J., Simmons, E., \& Mayer, K. H. (2005). HIV/AIDS and the black church: What are the barriers to prevention services? Journal of the National Medical Association, 97(12), 1682-1685.

Valera, P., \& Taylor, T. (2011). "Hating the Sin but not the Sinner": A study about heterosexism and religious experiences among Black men. Journal of Black Studies, 42(1), 106-122. https://doi. org/10.1177/0021934709356385.

Warner, L. R., \& Shields, S. A. (2013). The intersections of sexuality, gender, and race: Identity research at the crossroads. Sex Roles, 68(11), 803-810. https://doi.org/10.1007/s11199-013-0281-4

Watkins, T. L., Simpson, C., Cofield, S. S., Davies, S., Kohler, C., \& Usdan, S. (2016a). The relationship between HIV risk, high-risk behavior, religiosity, and spirituality among Black men who have sex with men (MSM): An exploratory study. Journal of Religion and Health, 55(2), 535-548. https:// doi.org/10.1007/s10943-015-0142-2.

Watkins, T. L., Simpson, C., Cofield, S. S., Davies, S., Kohler, C., \& Usdan, S. (2016b). The relationship of religiosity, spirituality, substance abuse, and depression among Black men who have sex with men (MSM). Journal of Religion and Health, 55(1), 255-268. https://doi.org/10.1007/s1094 3-015-0101-y.

Wilson, P. A., Wittlin, N. M., Muñoz-Laboy, M., \& Parker, R. (2011). Ideologies of Black churches in New York City and the public health crisis of HIV among Black men who have sex with men. Global Public Health, 6(Suppl 2), S227-S242. https://doi.org/10.1080/17441692.2011.605068.

Wilson, P. A., Valera, P., Martos, A. J., Wittlin, N. M., Muñoz-Laboy, M. A., \& Parker, R. G. (2016). Contributions of qualitative research in informing HIV/AIDS interventions targeting Black MSM in the United States. The Journal of Sex Research, 53(6), 642-654. https://doi.org/10.1080/00224 499.2015.1016139

Winder, T. J. A. (2015). "Shouting it out": Religion and the development of Black gay identities. Qualitative Sociology, 38(4), 375-394. https://doi.org/10.1007/s11133-015-9316-1.

Publisher's Note Springer Nature remains neutral with regard to jurisdictional claims in published maps and institutional affiliations. 